\title{
Influence of transannular interaction over absorption and fluorescent properties of [2.2] paracyclophane and its phenyl derivatives
}

\author{
R.N. Nurmukhametov ${ }^{1}$, A.V. Shapovalov, ${ }^{2,}$, and D.Yu. Antonov ${ }^{2}$ \\ ${ }^{1}$ L. Ya. Karpov Institute of Physical Chemistry RAS, 105064 Moscow, Russia \\ ${ }^{2}$ A.N. Nesmeyanov Institute of Organoelement Compounds RAS, 119991 Moscow, Russia
}

\begin{abstract}
A significant bathochromic shift of the fluorescent and longwavelength absorption bands of [2.2] paracyclophane comparing to corresponding bands of alkyl-benzenes is due to a strong transannular interaction, resulting in formation of a principally new excited state of lower energy. It is concluded that the fluorescent levels for alkylbenzene excimers and for the macrocycle are of the same nature. Analysis of [2.2] paracyclophane mono- and diphenylderivatives spectra shows that their intensive absorption bands $(230-310 \mathrm{~nm})$ are originated from electron transitions of biphenyl groups and weak long wavelength absorption (310 $340 \mathrm{~nm})$ and fluorescent bands are governed by the same electron transitions between ground and excimer-like excited states as in the case of non-substituted macrocycle.
\end{abstract}

It is well known, that UV-irradiation of organic luminophores solutions (included solid solutions in polymer) initiates in these systems photophysical and photochemical processes, which are essentially depended on interactions between molecules in excited and nonexcited states. In one cases this leads to excited dimmers (excimers) formation, in the others - to concentration decay of fluorescence or photochemical reactions. For organic compounds these processes cause non-radiative degradation of singlet excited state, which leads to fluorescence efficiency decrease in comparing to the single molecules emission. To investigate how these factors influence on the fluorescence of organic molecules, systems that contain several fragments with $\pi$-electron system in the certain orientation to each other are commonly used. In case when two chromophoric groups are included into cycle and located at a low distance non-valent interaction (so called "transannular interaction") originates. Due to this interaction absorption and luminescent properties of the macrocycles are considerably different in comparison with the properties of their non-cycled analogues. [2.2] Phanes are compounds possessed two chromophoric groups at a distance about $3 \AA$ in "face-to-face" position by means of ethylene linkages. Such macrocycles let us to investigate dimmer or excimer emission of sandwich-like compounds and polymers.

It is estimated that the main reason of significant bathochtomic shift of [2.2] paracyclophane ([2.2] PCP) fluorescent and long-wavelength absorption bands compared with the corresponding bands for molecules containing non-conjugated benzene chromophores is a strong transannular interaction between $\pi$-orbitals of closely spaced benzene rings of the macrocycle. This interaction leads to formation of principally new

* Corresponding author: Shapovalov-alex@yandex.ru 
excited state of lower energy. It is concluded that the fluorescent state for the macrocycle and for alkyl-benzenes excimers are of the same nature. It is supported by the fact that [2.2] PCP characterizes by very low quantum yields $(\sim 1 \%)$ and fluorescence lifetimes $(\sim 10 \mathrm{~ns})$ as well as excimer emission of benzene and its methyl derivatives (corresponding parameters for alkyl-benzenes molecular fluorescence are markedly greater: $\sim 10 \%$ and $\sim 25 \mathrm{~ns}$ ). Another characteristic feature of [2.2] PCP and alkyl-benzenes excimer fluorescence is their featureless emission bands even at low temperatures. The [2.2] PCP fluorescence band location at longer wavelength compared with polystyrene excimer emission band is explained by the fact that macrocycle HOMO energy level is situated higher than that of alkyl-substituted benzenes (the difference between [2.2] PCP and $p$ xylene ionization potentials is equal to $0.34 \mathrm{eV}, 2720 \mathrm{~cm}^{-1}$, which agrees well with the mentioned bathochromic shift $-3130 \mathrm{~cm}^{-1}$ ) [1]. That is why no anomalously large Stokes shift (one of the excimer emission characteristic signs) for [2.2]PCP fluorescence band can be observed.

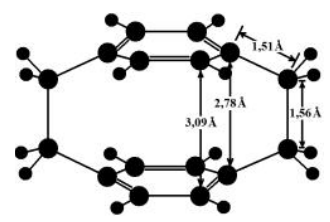

a)

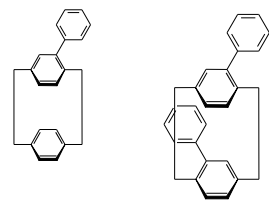

II

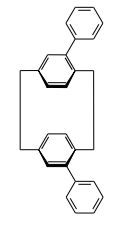

III

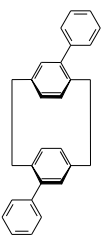

IV

Fig. 1. [2.2] PCP molecular structure (a) and its derivatives (I - IV).

[2.2] PCP phenyl derivatives I - IV are interesting objects for electron spectroscopy because the paracyclophane chromophore is retained in them and they also contain other groups with $\pi$-conjugated system. Analysis of I - IV absorption spectra shows that they are determined by two types of chromophores: biphenyl and paracyclophane. It is shown that their weak long wavelength absorption bands at $310-340 \mathrm{~nm}$ are governed by paracyclophane chromophore and strong bands at $230-310 \mathrm{~nm}$ originate from electronic transitions of biphenyl groups. It is supposed that observed difference of isomeric macrocycles II - IV strong bands is caused by different resonance interaction between electron oscillators (transitions) of the two biphenyl chromophores leading to Davydov splitting of their excited states [2]. The magnitude of the splitting is inversely proportional to the distance between the molecules. This distance in single crystal of benzene is slightly greater than that between the chromophores in II - IV, therefore resonance interaction in macrocycles is expected to be more pronounced. It is demonstrated that electronic transition energy of the paracyclophane chromophore changes little upon phenyl substitution: fluorescence spectra of I - IV solutions at 298 and $77 \mathrm{~K}$ exhibit featureless bands with shapes similar to the band of [2.2] PCP solution, slightly shifted to it bathochromically for $10-20 \mathrm{~nm}$ (like their long wavelength absorption bands) [3]. I - IV quantum yields are 2 3 times higher than that of the non-substituted compound and their fluorescence lifetimes are approximately the same. It also supports that their $\mathrm{S}_{1} \leftrightarrow \mathrm{S}_{0}$-transition is determined by paracyclophane chromophore. It can be concluded that long-wavelength absorption and fluorescent properties of [2.2] PCP phenyl derivatives are determined by excimer-like excited state that essentially forms due to transannular interaction (as for non-substituted [2.2] PCP). These results will facilitate interpretation of other cyclophanes $\pi$-electron features which are essential for understanding the nature of photophysical and photochemical processes in polychromophoric systems.

\section{References}

1. R.N. Nurmukhametov, A.V. Shapovalov, A.M. Sergeev, J. Appl. Spectr. 81, 49 (2014)

2. A.S. Davydov, Theory of Molecular Excitons (Nauka, Moscow, 1968) (in Russian)

3. R.N. Nurmukhametov et al., J. Appl. Spectr. 83, 27 (2016) 